common problem involves definitions. For example, the act of crawling by a dystrophic person does not make crawling a pathological trait. The act is a necessary reversion to a simpler mode of locomotion. Likewise, memory failure in senile disorders is revealed by circumlocution but circumlocutory speech itself is not abnormal. In fact, circumlocution proves that only a name and not a memory is lost. These signs of dementia appear with the loss of higher, more complex functions, unmasking simpler, surviving neurolinguistic mechanisms. The memory tricks senile individuals employ are wrongly labelled pathological when the same tactics in normals are called mnemonic aids. Indeed, portmanteau words in name lists (e.g. horseshoe) are actually constructed by circumlocutory invention. Certain colour terms (e.g. orange) represent deliberate adjective/noun blurring to serve mnemonic convenience.

Important clues to normal brain function may be overlooked if the data on language disorders are wrongly interpreted. We may ask if the naming of orphans in alphabetical sequence in Charles Dickens' Oliver Twist is evidence of indifferent efficiency or compensation for a defective memory? What we regard as learning tricks may be partly imposed on the process of memorisation by the brain itself. The neuronal substrate for memory must be far older than the mammalian cortex and it should be expected to have properties that shape the storage of inflowing data. Serially arranged elements such as the days of the week, the colours in the solar spectrum, and number systems seem to be more strongly embedded in the memory than loosely linked items. This is probably because a lost item can be recovered from adjacent elements by some rules of continuity. It is probably safe to assume that sequencing is a primitive neurolinguistic device for grouping similar data in close synaptic proximity. If data are stored in a manner to facilitate recovery of the message with minimum loss, then we are following a process akin to the error-resistant coding used in computer science (Goode \& Machol, 1957; Turner, 1968). The error-resistant Gray code is designed so that adjacent sequence items differ in the smallest possible manner. If each item is represented by a string of symbols (or neuronal pulses), and one symbol is lost during decoding, then the message error can still escape being total nonsense. A message string for the number " 6 " might be decoded after an error as "7", by use of a Gray code, and this may be allowable if we are trying to remember a birthday of a friend. If " 6 " prints out as " 16 " or " 61 " the number is no longer of value. The amino acid code for proteins and the nucleic acid code for the genetic material in the cell appear to be based on a molecular Gray code (Swanson, 1984). We may be missing similar insights on brain and memory functions by labelling inherent qualities as pathological traits rather than glimpses of normally functioning primitive mechanisms substituting for impaired higher language processes. The meaning of circumlocution, repetition, echolalia, etc. may need reexamination.

Research Laboratory 151-F,

ROBERT E. KUTTNER

University of Health Sciences/Chicago Medical School at N. Chicago VA Medical Center,

N. Chicago, Illinois 60064

References

GoODE, H. H. \& MACHOL, R. E. (1957) System Engineering. London: McGraw-Hill.

Swanson, R. (1984) A unifying concept for the amino acid code. Bulletin of Mathematical Biology, 46, 187-203.

Turner, J. F. (1968) Digital Computer Analysis. Columbus, Ohio: C. E. Merrill.

\section{REVERSIBLE DEMENTIA AND DEPRESSION}

\section{DEAR SIR,}

Several recent letters have commented on our article 'Criteria for Diagnosing Reversible Dementia Caused by Depression: Validation by 2-year Follow-up.' We agree with Dr Colgan (Journal, August, 1984, 145, 213-214), and stated in the paper, that a careful clinical examination is the best means for properly recognising such patients. As we noted, however, several studies have demonstrated that patients have been misdiagnosed as irreversibly demented rather than depressed in the past. We also agree with Dr Mahendra (Journal, August, 1984, 145, 213) and noted in the paper that underlying brain dysfunction could be giving rise to the symptoms of mood disorder and cognitive disorder. We cited Post as having suggested this 15 years ago.

Doctors Spagnoli and Williams (Journal, September 1984, 144, 339-340) question our use of the term "predictive value". This term is usually applied to assess screening tools and is affected by the prevalence of the disorders being studied. We used the term in a more constricted way to demonstrate the relationship between certain depressive symptoms and the reversible dementia syndrome of depression as compared to dementia. We wanted to emphasise that most symptoms used to identify depressive illness do identify persons with cognitive disorder who improve cognitively when the depression is treated. We did not 
comment in the paper but it is of note that not all symptoms associated with depression distinguish between the irreversible dementias and this reversible syndrome.

\section{Peter V. Rabins}

Gerald NeSTADT

The Johns Hopkins Hospital,

The Henry Phipps Psychiatric Clinic,

600 N. Wolfe Street, Baltimore, Maryland 21205

\section{ELECTROSTIMULATION AND OPIATE} WITHDRA WAL

DeAR Sir,

The paper by Gossop et al (Journal, February 1984, 144, 203-208), which has just been brought to my attention by Dr Connell, comparing electrostimulation with oral methadone in opioid withdrawal, contains several theoretical and factual errors. The techniques as described are certainly not, as claimed, "the same as the 'NET' (neuro-electric therapy) used by Patterson".

Moreover, I have never advocated "acupuncture techniques", also claimed by the authors, because acupuncture, as practised by experts in Hong Kong and China, had little or no effect on drug addiction. Electro-acupuncture was simply a means by which we serendipitously discovered that electric signals were a significant therapeutic tool.

One fault in design lies in comparing drug-free patients with those receiving a potent opioid (methadone). A more valid comparison would be 10 days after each group had completed the withdrawal treatment, despite the authors' admission of design "weakness" in comparing a 10 day treatment (NET) with a 21 day treatment (methadone withdrawal-MW). The 10 day posttreatment results show a distinct advantage in the symptoms graph (page 206) for NET as against MW.

One of the greatest benefits of NET is the reduction of the chronic withdrawal period from many months-up to 18 months in methadone addiction (Cushman, 1978) - to 1 to 2 weeks, occasionally up to 4 weeks, after commencement of NET (Patterson, 1983).

Further, only 8 cases out of 24 completed Gossop's study. In my detailed examination of the withdrawal symptomatology in 102 consecutive cases treated over one year, there was only one drop-out before completion of the 10 days NET (Patterson, 1984). Also, according to the authors, there was no objective reporting of symptomatology by staff. In my much larger study, although there was no comparison group, symptoms and signs were recorded 4 times daily by trained nurses for the first 6 days and twice daily for the next 4 days. This was in addition to once daily selfreporting by the patients. It is well known that addicts exaggerate their symptoms, yet Gossop's study bases its findings on such unreliable data.

"All the subjects in the study used a variety of other drugs on an occasional basis". Apparently no effort was made to compare patients using the same drug or drug combination, not is it stated how many in each group were using each different drug.

One of the basic tenets of NET is that the current frequency has to be altered for each different group of psychoactive drugs and occasionally for different drugs within the same group, the problem becoming much more complicated with drug combinations. There is no evidence from the article that this fact, although repeatedly mentioned in my several publications, has been grasped by the participants in this study. It is not surprising that they had so little success if, for example, they used "for opiates and benzodiazepines $400 \mathrm{~Hz}$ ".

Their report provides no follow-up although it was completed in June 1982 and not published until 1984. I have twice published my follow-up results (Patterson, 1984), the second including patients treated up to 8 years previously.

Finally, the letter from Dr E. P. Larkin (Journal, June 1984, 144, 670-671) suggesting using NET and MW together ignores the findings of Man and Chuang, quoted in Gossop's article, in which 30 days of MW was given to both control and study groups. They themselves dismissed the study as "meaningless" because 83 per cent were found to be using drugs illicitly although in-patients-another factor which Gossop and colleagues appear not to have investigated. In my 12 years' experience, there has been consistent clinical evidence that concurrent administration of drugs diminishes the beneficial effects of NET.

\section{Margaret A. Patterson}

102 Trevelyan Road,

London SW17 9LN

References

Cushman, P. (1978) Detoxification of methadone maintained patients. In Drug Abuse: Modern Trends, Issues and Perspec. tives (eds. A. Schecter, H. Alksne \& E. Kaufman). New York: Marcel Decker.

Patterson, M. (1983) Getting Off The Hook. Addictions Can Be Cured by NET (Neuro-electric Therapy). Wheaton, Illinois: Harold Shaw.

- Firth, J. \& Gardiner, R. (1984) Treatment of drug, alcohol and nicotine addiction by neuro-electric therapy: analysis of results over 7 years. Journal of Bio-electricity, 3, 193-221. 